\title{
PROPAGATION OF TE WAVES IN NONLINEAR WAVEGUIDE
}

\author{
G. ŻEGLIŃsKi ${ }^{a}$ AND M. WABIA ${ }^{b}$ \\ ${ }^{a}$ Department of Optical Telecommunication and Photonics \\ Technical University of Szczecin, 26 Kwietnia 10, 71-062 Szczecin, Poland \\ ${ }^{b}$ Institute of Physics, Technical University of Szczecin \\ Al. Piastów 48, 70-310 Szczecin, Poland
}

\begin{abstract}
In the paper numerical calculations of the results of propagation in a nonlinear planar waveguide are performed. A type of nonlinearity is the Kerr nonlinearity. The wave equation, the solution of wave equation are shown, the dispersion equation, and the numerical solution of dispersion equation are analysed.
\end{abstract}

PACS numbers: 42.65.-k, 42.65.Tg

\section{Introduction}

Propagation in linear planar waveguides is a well-known problem, whereas propagation in nonlinear waveguides was considered only in particular cases. That is the case of the nonlinear waveguide with a linear film on a nonlinear substrate [1], then the linear film surrounded by the nonlinear substrate and cover [2-7], the nonlinear film surrounded by linear isotropic media [8-15]. In the paper the analysis of electromagnetic field in the nonlinear planar waveguide is undertaken, where all media have the Kerr type of nonlinearity.

The mathematical analysis of the electromagnetic field in the nonlinear waveguide is more complicated than the nonlinear interface because of:

- two interfaces and three nonlinear media exist, so three nonlinear waves can appear,

- the nonlinear wave equation can have a more general form than in the case of the nonlinear interface. 


\section{The model of nonlinear planar waveguide}

The nonlinear waveguide is a structure of the consisting three layers presented in Fig. 1. In this structure we have

- layer 1 - cover $(x>l)$,

- layer 2 - film $(-l<x<l)$,

- layer 3 - substrate $(x<-l)$.

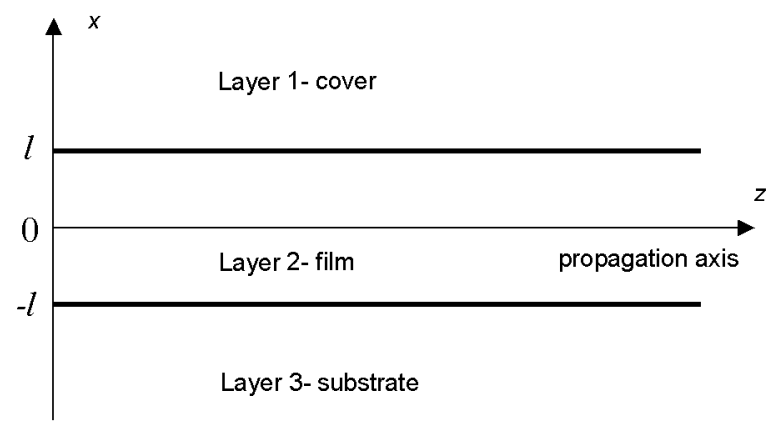

Fig. 1. The model of the nonlinear planar waveguide.

Each layer is the Kerr nonlinear medium which is described by the uniaxal permittivity tensor ${ }^{j} \widehat{\varepsilon}(j=1,2,3)$

$$
j \widehat{\varepsilon}=\left(\begin{array}{ccc}
{ }^{j} \varepsilon_{11} & 0 & 0 \\
0 & { }^{j} \varepsilon_{22} & 0 \\
0 & 0 & { }^{j} \varepsilon_{33}
\end{array}\right) .
$$

We assume that only the tensor component ${ }^{1} \varepsilon_{22}$ depends on the perpendicular component of electric field $E_{y j}$. The tensor components (1) have then the following form:

$$
\begin{aligned}
& j_{\varepsilon_{11}}={ }^{j} \varepsilon_{\varepsilon_{33}}=j_{\varepsilon}, \\
& j_{\varepsilon_{22}}=j_{\varepsilon_{22}}+\alpha_{j}\left|E_{y j}\right|^{2},
\end{aligned}
$$

where ${ }^{1} \varepsilon, j_{\varepsilon_{1}}$ - the linear permittivities of media, $\alpha_{j}$ - the nonlinear factor in medium, $E_{y j}$ - the perpendicular component of the electromagnetic field.

The subject of the further investigation will be TE field in the nonlinear waveguide structure using the approximations $(2-3)$.

\section{Maxwell equations and the wave equation}

The electrical field vector of TE-waves has only one nonzero component, for instance $E_{y}$. The magnetic field strength vector components for this sort of waves are connected with $E_{y}$ by the Maxwell equations.

For the planar waveguide, which is described by the dielectric tensor with nonlinearity (2-3), the Maxwell equations for TE polarisation can be written as

$$
\frac{\partial E_{y j}}{\partial z}=-\mathrm{i} \omega \mu_{0} H_{x j}
$$




$$
\begin{aligned}
& \frac{\partial E_{y j}}{\partial x}=\mathrm{i} \omega \mu_{0} H_{z j}, \\
& \frac{\partial H_{x j}}{\partial z}-\frac{\partial H_{z j}}{\partial x}=-\mathrm{i} \omega \varepsilon_{0}{ }^{j} \varepsilon_{22} E_{y j},
\end{aligned}
$$

where $\varepsilon_{0}$ and $\mu_{0}$ - the permivitty and the permeability of free space, respectively, $j=1,2,3$ is the index of the region of the waveguide structure.

Due to the translation symmetry along the $z$-axis the $y$-component of the electric field $E_{y}(\omega, x, z)$ can be chosen as

$$
E_{y j}(x, z, t)=E_{y j}(x) \exp \left[\mathrm{i}\left(k_{0} h z-\omega t\right)\right],
$$

where $E_{j}(x)$ - the amplitude of electric field mode in $j$-layer, $h$ - the constant of the propagation, $k_{0}=\omega / c$. This component describes the TE wave propagation along the $z$ direction.

In the assumed model of the field propagation, the wave equation in the $j$ region of the nonlinear waveguide structure can be obtained from the Maxwell equations (4-6) and with the uniaxial approximation (1-3) it takes the following form:

$$
\frac{\mathrm{d}^{2} E_{y}}{\mathrm{~d} x^{2}}-k_{0}^{2}\left(h^{2}-\varepsilon\right) E_{y}+k_{0} \alpha\left|E_{y}\right|^{2} E_{y}=0 .
$$

For $\alpha_{j}>0$ the solution of the wave equation is given by the elliptic function of Jacobi cosine

$$
E_{y}=p_{j} \operatorname{cn}\left[q_{j} k_{0}\left(x-x_{0 j}\right) \mid m_{j}\right],
$$

where

$$
\begin{aligned}
& p_{j}^{2}=\frac{h^{2}-j_{\varepsilon}+\left[\left(h^{2}-j_{\varepsilon}\right)^{2}+2 \alpha_{j} C_{1} / k_{0}^{2}\right]^{1 / 2}}{\alpha_{j}}, \\
& q_{j}^{2}=\left[\left(h^{2}-j_{\varepsilon}\right)^{2}+2 \alpha_{j} C_{1} / k_{0}^{2}\right]^{1 / 2}, \\
& m_{j}=\frac{\alpha_{j} p_{j}^{2}}{2 q_{j}^{2}}
\end{aligned}
$$

$C_{1}, x_{0 j}$ - integral constants. For $C_{1}=0$ and $m=1$ Jacobi cosine has a form of decay solution

$$
E_{y}=p_{j}\left\{\cosh \left[q_{j} k_{0}\left(x-x_{0 j}\right)\right]\right\}^{-1},
$$

where

$$
\begin{aligned}
& p_{j}=\sqrt{\frac{2\left(h^{2}-j_{\mathcal{E}}\right)}{\alpha_{j}}}, \\
& q_{j}=\sqrt{h^{2}-{ }^{j_{\mathcal{E}}}} .
\end{aligned}
$$

In the model of the field propagation in the nonlinear planar waveguide, we seek for the solutions of the field equation (8), which have the following character: - in layer 1 (cover), the decay solution - $\cosh ^{-1}$,

- in layer 2 (film), the periodical solution - $\mathrm{cn}(m \mid u)$,

- in layer 3 (substrate), the decay solution $-\cosh ^{-1}$. 


\section{The dispersion equation}

Continuity conditions at interfaces between layers $(x= \pm l)$ require continuity of the tangent components of the electrical and magnetical fields, $E_{y}(x)$ and $H_{z}(x)$

$$
\begin{aligned}
& E_{y 2}(-l)=E_{y 3}(-l), \\
& \left.\frac{\mathrm{d}\left(E_{y 2}(x)\right)}{\mathrm{d} x}\right|_{x=-l}=\left.\frac{\mathrm{d}\left(E_{y 3}(x)\right)}{\mathrm{d} x}\right|_{x=-l}, \\
& E_{y l}(l)=E_{y 2}(l), \\
& \left.\frac{\mathrm{d}\left(E_{y 1}(x)\right)}{\mathrm{d} x}\right|_{x=-l}=\left.\frac{\mathrm{d}\left(E_{y 2}(x)\right)}{\mathrm{d} x}\right|_{x=-l} .
\end{aligned}
$$

Using the solution (13) for a substrate and a cover and using the solution (9) for a film, the continuity conditions give a dispersion equation for the nonlinear waveguide

$$
\begin{aligned}
& \left(h^{2}-\varepsilon_{2}\right)^{2}\left(A_{1}^{2}-B_{1}^{2}\right)+2\left(h^{2}-\varepsilon_{2}\right) B_{1} C_{1} \\
& \quad+2 \alpha_{2}\left(\varepsilon_{2}-\varepsilon_{1}\right) A_{1}^{2} E_{01}^{2}+\alpha_{2}\left(\alpha_{1}-\alpha_{2}\right) A_{1}^{2} E_{01}^{4}-C_{1}^{2}=0,
\end{aligned}
$$

where

$$
\begin{aligned}
& A_{1}=2\left(\varepsilon_{2}-\varepsilon_{1}\right)+\left(5 \alpha_{1}-4 \alpha_{2}\right) E_{01}^{2}, \\
& B_{1}=2\left(\varepsilon_{2}-\varepsilon_{1}\right)+\left(\alpha_{1}-2 \alpha_{2}\right) E_{01}^{2}, \\
& C_{1}=2\left(\varepsilon_{2}-\varepsilon_{1}\right) E_{01}^{2}+\left(\alpha_{1}-\alpha_{2}\right) E_{01}^{4} .
\end{aligned}
$$

Equation (20) is strictly connected with Eq. (25) by the condition $E_{01}=E(x=l)$. The constant $E_{01}$ is also univocally dependent on the assumed constant $E_{0}=E_{02}$, which describes the value of electric field strength on the lower interface $x=-l$.

The solution of the dispersion equation is

$$
h= \pm \sqrt{\varepsilon_{2}+\frac{-2 B_{1} C_{1} \pm \sqrt{2 A_{1}^{2} C_{1}\left[2 C_{1}-\left(A_{1}^{2}-B_{1}^{2}\right)\right]}}{2\left(A_{1}^{2}-B_{1}^{2}\right)}} .
$$

$E_{01}$ can be calculated from

$$
\begin{gathered}
\varepsilon_{2}+\frac{-2 B_{1} C_{1} \pm \sqrt{2 A_{1}^{2} C_{1}\left[2 C_{1}-\left(A_{1}^{2}-B_{1}^{2}\right)\right]}}{2\left(A_{1}^{2}-B_{1}^{2}\right)}= \\
\varepsilon_{3}+\frac{-2 B_{2} C_{2} \pm \sqrt{2 A_{2}^{2} C_{2}\left[2 C_{2}-\left(A_{2}^{2}-B_{2}^{2}\right)\right]}}{2\left(A_{2}^{2}-B_{2}^{2}\right)}
\end{gathered}
$$

where

$$
\begin{aligned}
& A_{2}=2\left(\varepsilon_{3}-\varepsilon_{2}\right)+\left(5 \alpha_{2}-4 \alpha_{3}\right) E_{02}^{2}, \\
& B_{2}=2\left(\varepsilon_{3}-\varepsilon_{2}\right)+\left(\alpha_{2}-2 \alpha_{3}\right) E_{02}^{2}, \\
& C_{2}=2\left(\varepsilon_{3}-\varepsilon_{2}\right) E_{02}^{2}+\left(\alpha_{2}-\alpha_{3}\right) E_{02}^{4} .
\end{aligned}
$$




\section{Calculations}

For the calculation purpose, we take the length of wave $\lambda=0.633 \mu \mathrm{m}$ and three nonlinear Kerr media, which built a nonlinear waveguide structure, with their guiding layer of thickness $2 l=0.7 \mu \mathrm{m}$ and with the following values of refractive indexes [13-15]:

- cover - crystal MBBA $\left(n_{01}=1.55, n_{21}=10^{-9} \mathrm{~m}^{2} / \mathrm{W}\right)$,

- film - crystal YAG $\left(n_{02}=1.83, n_{22}=3 \times 10^{-9} \mathrm{~m}^{2} / \mathrm{W}\right)$,

- substrate - crystal MBBA $\left(n_{01}=1.55, n_{21}=10^{-9} \mathrm{~m}^{2} / \mathrm{W}\right)$,

where $n_{01}, n_{02}, n_{21}, n_{22}$ are the linear and nonlinear parts of the refractive indexes of the crystals MBBA and YAG.

The picture of electromagnetic field in nonlinear wa veguide shows the profile of electric field distribution for a defined mode for a waveguide. The calculation for the parameters assumed above gives the profile of $\mathrm{TE}_{0}$ illustrated in Figs. 2 and 3 .

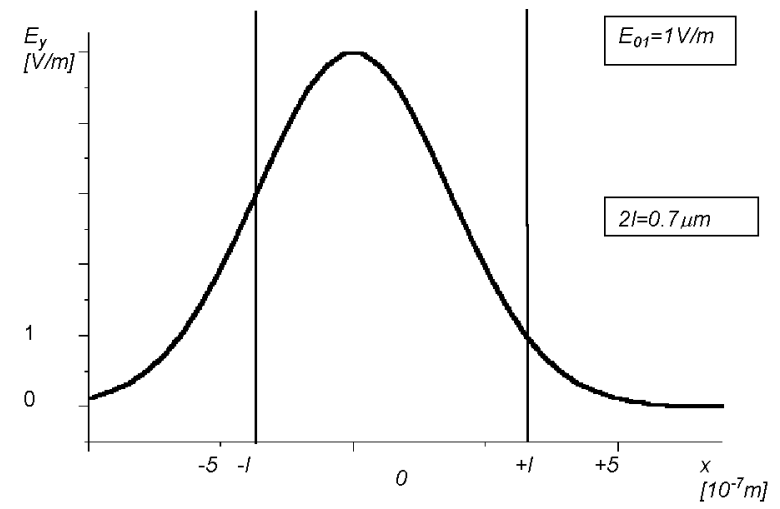

Fig. 2. The intensity profile of $\mathrm{TE}_{0}$ electric field mode in the nonlinear planar waveguide for $E_{01}=1 \mathrm{~V} / \mathrm{m}, 2 l=0.7 \mu \mathrm{m}$.

As can be seen an electric field maximum of the basic mode is situated in the film and asymmetrically to the symmetry axis of the film. In the region of a substrate and a cover the field decays rapidly. Such asymmetry is caused by electric field of a very big value, which is encountered in a nonlinear medium. The electric field $\mathrm{E}_{02}$ generated on one interface excites a nonlinear polarization of a medium, and as a result, an appropriate value of the strength of the electric field $\mathrm{E}_{01}$ on the second interface. The value of strength $E_{01}$ varies from $E_{02}$, which is shown in the expressions (21-28). All that creates an asymmetric waveguide, in spite of the same material layers of a substrate and a cover. Such a symmetry causes "degeneration" of the field guided in a waveguide. This means pushing in of the field or its transformation into surface wave guided by only one interface. Nonlinearity of medium near the one of the interfaces became so strong that the electromagnetic field creates its own canal along the interface and propagates itself as the surface wave in that canal. In the case showed in Figs. 2 and 3 such situation 


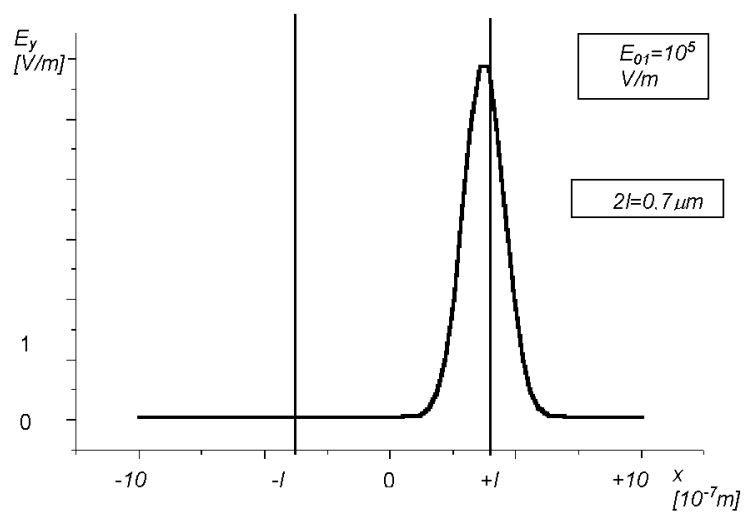

Fig. 3. The intensity profile of $\mathrm{TE}_{0}$ electric field mode in the nonlinear planar waveguide for $E_{01}=10^{5} \mathrm{~V} / \mathrm{m}, 2 l=0.7 \mu \mathrm{m}$.

takes place on the upper interface $x=l$ and for the modes with a great value of the propagation constant $h$.

A similar situation is already mentioned in the literature [16] in the case of a nonlinear waveguide structure consisting of a linear substrate, a linear film and a nonlinear cover. For great values of electric field, the mode guided in a film is caught by the interface between a cover and a film, and "degenerates" into a surface wave guided by that interface.

It is known that for exciting an electromagnetic field in nonlinear structures, a certain threshold power is necessary. From that reason the power guided in the waveguide is also an important parameter.

The flow of power for harmonic field is given by an average value of the Poynting vector, and the power being transported by waves in the direction of the waveguide axis can be obtained by integration of this value into a direction perpendicular to the waveguide axis. As a result, the power guided in $j$ direction of the waveguide region is given by the following expressions:

$$
\begin{aligned}
& P_{1}=\frac{k_{0} h}{2 \omega \mu_{0}} \int_{l}^{\infty}\left|E_{y 1}(x)\right|^{2} \mathrm{~d} x, \\
& P_{2}=\frac{k_{0} h}{2 \omega \mu_{0}} \int_{-l}^{+l}\left|E_{y 2}(x)\right|^{2} \mathrm{~d} x, \\
& P_{3}=\frac{k_{0} h}{2 \omega \mu_{0}} \int_{-\infty}^{-l}\left|E_{y 3}(x)\right|^{2} \mathrm{~d} x,
\end{aligned}
$$

The total power guided by the nonlinear TE waves in a waveguide is the sum of the power guided in particular regions of the waveguide

$$
P_{c}=\sum_{j=1}^{3} P_{j},
$$

and is a function of the mode index $h$. 
The results of the power guided in particular regions of the waveguide is presented in Figs. 4 and 5. In these figures it is easy to see that the power guided by the $\mathrm{TE}_{0}$ mode in a substrate and a cover of a nonlinear waveguide structure is a function of the propagation constant $h$.

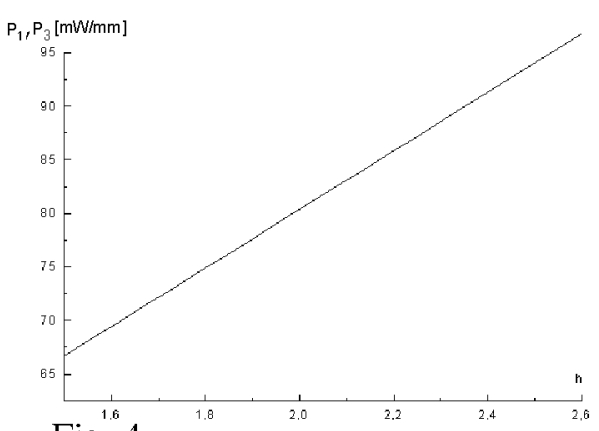

Fig. 4

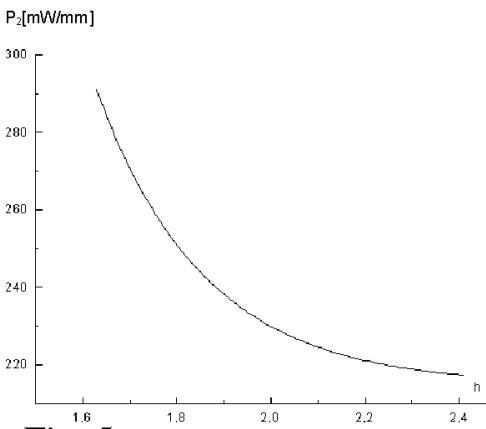

Fig. 5

Fig. 4. The power $\mathrm{TE}_{0}$ mode dependence of the propagation constant in media 1, 3 .

Fig. 5. The power $\mathrm{TE}_{0}$ mode dependence of the propagation constant in medium 2.

From Fig. 5 one can see that for exciting the electromagnetic field in the considering nonlinear waveguide structure, a certain critical power is needed, below which no exciting of a guided nonlinear wave (NLGW) in the waveguide is possible.

\section{Conclusions}

On the basis of the reached results we can state that:

a) In a planar waveguide structure consisting of three focusing nonlinear layers, the excitement and the guiding of the nonlinear TE field modes (NLGW) is possible.

b) For exciting the nonlinear modes, guided in a layer (NLGW), a certain threshold power is necessary (Fig. 5).

c) For great values of the electric field, a guided wave (NLGW) "degeneration" can happen either by its transformation into two surface waves (NLSW) guided by two interfaces, or into one surface wave on the one of the interfaces.

\section{References}

[1] D. Michalache, H. Totia, Solid State Commun. 54, 175 (1985).

[2] N.N. Akhmediev, Sov. Phys. JETP 56, 299 (1982).

[3] F. Lederer, U. Langbein, H.E. Ponath, Appl. Phys. B 31, 69 (1983).

[4] A.D. Boardman, P. Egan, V. Shivarova, Appl. Sci. Res. 41, 343 (1984).

[5] G.I. Stegeman, J.T. Chiwell, S.D. Smith, IEEE J. Quantum Electron. QE-21, $774(1985)$. 
[6] A.D. Boardman, P. Egan, J. Phys. Colloq. C 5, 291 (1984).

[7] F. Lederer, U. Langbein, H.E. Ponath, Appl. Phys. B 31, 187 (1983).

[8] G.S. Stegeman, C.T. Seaton, J.T. Chilwell, S.D. Smith, Appl. Phys. Lett. 44, 830 (1984).

[9] N.N. Akhmediev, K.O. Boltar, V.M. Eleonskii, Opt. Spectrosc. 53, 906 (1982).

[10] U. Langbein, F. Lederer, H.E. Ponath, Opt. Commun. 46, 167 (1983).

[11] A.D. Boardman, P. Egan, IEEE J. Quantum Electron. QE-22, 319 (1986).

[12] D. Mihalache, Solid State Commun. 58, 125 (1986).

[13] A.D. Boardman, P.E. Egan, F. Lederer, U. Langbein, D. Michalake, in: Nonlinear Surface Electromagnetic Phenomena, Eds. H.E. Ponath, G.I. Stegeman, North-Holland, Amsterdam 1991, Ch. 2.

[15] A.D. Boardman, P.E. Egan, T. Twardowski, M. Wilkins, in: Nonlinear Waves in Solid State Physics, Ed. A.D. Boardman, NATO Advanced Study Institute, Vol. 247, Series B: Physics, Plenum Press, New York 1992, Ch. 1.

[16] E. Wright, G.I. Stegeman, in: Anisotropic and Nonlinear Optical Waveguides, Eds. C.G. Someda, G. Stegeman, Elsevier Science Pub., New York 1992, p. 117. 